\title{
Power-law Tails from Dynamical Comptonization in Converging Flows
}

\author{
Roberto Turolla ${ }^{1}$, Silvia Zane ${ }^{2}$ and Lev Titarchuk $k^{3,4}$
}

\begin{abstract}
The effects of bulk motion comptonization on the spectral formation in a converging flow onto a black hole are investigated. The problem is tackled by means of both a fully relativistic, angle-dependent transfer code and a semi-analytical, diffusion-approximation method. We find that a power-law high-energy tail is a ubiquitous feature in converging flows and that the two approaches produce consistent results at large enough accretion rates, when photon diffusion holds. Our semi-analytical approach is based on an expansion in eigenfunctions of the diffusion equation. Contrary to previous investigations based on the same method we find that, although the power-law tail at really large energies is always dominated by the flatter spectral mode, the slope of the hard X-ray portion of the spectrum is dictated by the second mode and it approaches $\Gamma=3$ at large accretion rate, irrespective of the model parameters. The photon index in the tail is found to be largely independent on the spatial distribution of soft seed photons when the accretion rate is either quite low ( $\lesssim 5$ in Eddington units) or sufficiently high $(\gtrsim 10)$. On the other hand, the spatial distribution of source photons controls the photon index at intermediate accretion rates, when $\Gamma$ switches from the first to the second mode. Our analysis confirms that a hard tail with photon index $\Gamma<3$ is produced by the up-scattering of primary photons onto infalling electrons if the central object is a black hole.
\end{abstract}

Subject headings: accretion, accretion disks — black hole physics — radiation mechanisms: non-thermal — radiative transfer

\footnotetext{
${ }^{1}$ Department of Physics, University of Padova, via Marzolo 8, 35131 Padova, Italy; turolla@pd.infn.it

${ }^{2}$ Mullard Space Science Laboratory, University College London, Holmbury St. Mary, Dorking, Surrey, RH5 6NT, UK; sz@mssl.ucl.ac.uk

${ }^{3}$ George Mason University/Center for Earth Observing and Space Research, Fairfax, VA 22030 and US Naval Research Laboratory, Code 7620, Washington, DC 20375-5352, USA; lev@xip.nrl.navy.mil

${ }^{4}$ NASA/Goddard Space Flight Center, Greenbelt MD 20771, USA; lev@lheapop.gsfc.nasa.gov
} 


\section{Introduction}

The idea that photons may change their energy in repeated scatterings with cold electrons in a moving fluid has been suggested more than 20 years ago by Payne \& Blandford (1981) and Cowsik \& Lee (1982). This process, often refereed to as dynamical (or bulk) Comptonization, is completely equivalent to Comptonization by hot electrons once the thermal velocity is replaced by the bulk velocity v. However, as already noted by Cowsik \& Lee and Payne \& Blandford, it has to be $\nabla \cdot \mathbf{v} \neq 0$, like in a converging flow, for the mechanism to produce a full-fledged effect. If a photon interacts with electrons moving at uniform speed its energy is boosted by a factor $\sim \gamma^{2}=\left(1-v^{2}\right)^{-1}$, independently of the number of scatterings. On the other hand, in a flow where $\nabla \cdot \mathbf{v} \neq 0$ a photon typically scatters on electrons with different velocity and the change of the local rest frame introduces a differential effect. As shown by Payne \& Blandford (see also Nobili, Turolla \& Zampieri 1993), if monochromatic radiation with $\nu=\nu_{0}$ is injected at large Thomson depth in a spherical accretion flow the emergent spectrum is broad, shifted to $\nu>\nu_{0}$ and a typical power-law tail appears at high energies. For a power-law velocity law, $v \sim r^{-\beta}$, the photon spectral index is correlated to the velocity gradient and becomes 3 in free-fall. Dynamical Comptonization in the non-relativistic limit was also investigated by Schneider \& Bogdan (1989) and Mastichiadis \& Kylafis (1992), who considered a spherical flow onto a neutron star and found that the choice of the inner boundary condition affects the emerging power-law index substantially. The competing action of dynamical and thermal Comptonization in a semi-infinite medium at non-zero temperature was discussed by Colpi (1988) using the non-relativistic diffusion approximation. Titarchuk, Mastichiadis \& Kylafis (1996) extended Mastichiadis \& Kylafis' results including the effects of electron recoil and thermal motion. They demonstrated that the spectral power-law index goes to zero when the Thomson depth in the flow becomes very large.

The potential importance of dynamical Comptonization in connection with high-energy emission from compact X-ray sources has been recognized since the very beginning. However, it was not until the mid-90's that renewed interest in this topic was aroused by two facts: the observational evidence that X-ray spectra from Galactic black hole candidates (BHCs) may exhibit in the soft (high) state a power-law tail which extends up to hundreds of keV, and the introduction of the two-phase paradigm for accretion flows onto black holes (Chakrabarti \& Titarchuk 1995). In addition to standard, geometrically-thin accretion disks (SSDs, Shakura \& Sunyaev 1973), Chakrabarti \& Titarchuk (1995) presented arguments for the existence of a sub-Keplerian flow outside the disk. The popular advection-dominated accretion models (ADAFs), initially introduced for optically thin accretion flows onto black holes (see e.g. Narayan, Mahadevan \& Quataert 1999 for a review), are a particular class of sub-Keplerian flows, namely ADAFs are also nearly spherical and transonic close to the hole. In current 
models for BHCs, a sub-Keplerian component is thought to exist along with the SSD in the inner region of the accretion flow $(\lesssim 100$ gravitational radii) and it may provide the conditions for making dynamical Comptonization effective. Chakrabarti \& Titarchuk (1995), Titarchuk, Mastichiadis \& Kylafis (1996), and Ebisawa, Titarchuk \& Chakrabarti (1996) were the first to point out that in a realistic accretion flow the finite Thomson depth at the horizon, $\tau_{s, H}$ produces a power-law spectral index (which depends on $\tau_{s, H}$ ) flatter than 3 , in general agreement with the observed values (at least for $\dot{m} \lesssim 5$, as we will discuss later). Turolla, Zane, Zampieri \& Nobili (1996), solving analytically the general-relativistic moment equations, confirmed this result and provided a simple expression for the power-law index as a function of $\tau_{s, H}$ in a free-falling medium. Titarchuk, Mastichiadis \& Kylafis (1997), hereafter TMK97, considered a non-relativistic diffusion equation for the photon occupation number, including dynamical and thermal effects. They derived both numerical and (approximate) analytical solutions, and concluded that, while the spectral index depends on the location of the inner boundary and on the boundary conditions, it is not much sensitive to the spatial and energy distribution of the primary photons. Several numerical calculations, based on different approaches to the solution of the transfer problem, have been presented so far (relativistic moment method: Nobili, Turolla \& Zampieri 1993, Zampieri, Turolla \& Treves 1993; fully relativistic transfer equation: Zane, Turolla, Nobili \& Erna 1996, Titarchuk \& Zannias 1998; Monte Carlo simulations: Laurent \& Titarchuk 1999). Shrader \& Titarchuk (1998), following TMK97, were able to reproduce the observed X-ray spectrum of several BHCs, assuming that the accretion flow consists of two phases: a SSD and a radial component which replaces the disk close to the hole. Thermal photons emitted by the disk produce the observed soft emission at a few $\mathrm{keV}$ and are in part up-scattered by the inflowing electrons forming the power-law tail.

Numerical investigations based on Monte Carlo methods or $\Lambda$-iteration schemes typically fail in reproducing cases with relatively high $\dot{m}$, even well below the range where diffusion approximation holds. Analytical investigations, on the other hand, are supposed to reproduce the diffusion approximation limit, but suffer from a series of limitations imposed by the simplifying assumptions which need to be introduced. All of them are based on the solution of the first two moment equations neglecting, to a various extent, terms of higher order in $v$ and higher order moments. This approach is justified at large depth, but becomes questionable for $\tau_{s, H} \lesssim 1$. Moreover, analytical spectra have been often calculated for a monochromatic injection of photons at a given radius, so they fail to answer to the fundamental question of how the emergent spectrum depends on the primary photon distribution, both in energy and in space. An exception is the method presented by TMK97, that will be used in this investigation.

Quite surprisingly, while all studies seem to agree that a power-law tail forms at high 
energies in a converging flow, the derived values of the spectral index cover a considerable range. This can reflect either an inaccuracy of the methods or an intrinsic dependence of the spectral index on some (often implicit, even hidden) assumptions at the basis of the calculation, or both. In the attempt to clarify this point Papathanassiou \& Psaltis (2001) undertook a project aimed to a systematic exploration of the parameter space by means of the numerical integration of the relativistic transfer equation for a free-falling flow in a Schwarzschild spacetime. The results reported in their first paper are in agreement with the single model of Zane et al. (1996) but not with the analytical prediction of Turolla et al. (1996). The derived values of the spectral index are similar to those of Titarchuk \& Zannias and Laurent \& Titarchuk, but care must be taken in comparing this calculation to previous ones because Papathanassiou \& Psaltis (2001) use a different definition of $\tau_{s, H}$.

In this paper we clarify the role of the spatial distribution of the source of input photons. This has been often overlooked or misunderstood in the literature. In particular, we consider the difference between diffuse and spatially concentrated sources. We support our results with both a systematic numerical analysis and a semi-analytical calculation. The first is carried out by solving the transfer problem for a radially inflowing medium in a Schwarzschild spacetime using the code described in Zane, Turolla, Nobili \& Erna (1996). The latter is based on the method introduced by TMK97. Numerical models have been computed up to large enough values of $\tau_{s, H}$ to allow for a direct comparison with analytical, diffusionapproximation results. We show that the spatial distribution of seed photons is unimportant in fixing the power-law index both al small and large accretion rates $(\dot{m} \lesssim 5$ and $\dot{m} \gtrsim 15$

for a scattering-dominated accretion flow onto a black hole), while it influences the index at intermediate accretion rates. However, unless the source is extremely concentrated close to the horizon, the slope of high-energy tail does not depend much on the primary photon distribution. We also convincingly show that the photon index approaches 3 at large accretion rates, irrespective of the input parameters.

The plan of the paper is as follows. In $\S 2$ we introduce the numerical method for the solution of the relativistic kinetic equation and present the results of numerical calculations. Approximate analytical solutions in diffusion approximation are derived in $\S 3$ and compared with both our numerical models and results from previous investigations. Discussion and conclusions follow in $\S 4$.

\section{Numerical method and results}

A fairly general technique for the numerical solution of the relativistic transfer equation in spherical symmetry has been presented and discussed in Zane, Turolla, Nobili \& Erna 
(1996). The method makes use of the characteristics to reduce the comoving-frame transfer equation to an ordinary differential equation for the photon occupation number $f$ along the photon trajectories. To avoid any confusion, we stress that $f=f(r, \mu, E)$, the cosine of the angle between the photon and the radial directions $\mu$ and the photon energy $E$ are all measured by the comoving observer (LRF), while $r$ is the coordinate radius.

In the following we deal with a spherical flow in a Schwarzschild spacetime and use units in which $c=G=h=1$. The radial coordinate is in units of the Schwarzschild radius, $r_{S}=2 M$, where $M$ is the mass of the central source. For a Schwarzschild geometry Zane et al. derived simple analytical expressions for $\mu=\mu(r, b)$ and $E=E_{\infty} \epsilon(r, b)$, where $b$ is the ray impact parameter and $E_{\infty}$ the photon energy measured by an observer at rest at radial infinity. The transfer equation

$$
\frac{d f}{d r}=\frac{r_{S} \mathcal{G}(r, \mu, E, f)}{y E(\mu+v)},
$$

where $y=\gamma \sqrt{1-r_{S} / r}$ and $\mathcal{G}$ is the source term, is then solved for different values of the two parameters $b$ and $E_{\infty}$ to obtain the specific intensity $I=2 f E^{3}$. Ordinary $\Lambda$ iteration is used to reach convergence in case the source term contains scattering integrals. The radiation moments (mean intensity $J_{\nu}$, flux $H_{\nu}$ and pressure $K_{\nu}$, here $\nu$ is the photon frequency) are evaluated by direct numerical quadrature over angles of the specific intensity times the required power of $\mu$, at constant $E$ and $r$. We assume conservative (i.e. Thomson) scattering in the electron rest frame. Since we want to assess the effects of dynamical Comptonization, we ignore thermal motion and take the electron rest frame to coincide with the LRF (TMK97).

We assume free-fall so that $y v=r^{-1 / 2}$ which implicitly gives $v$ as a function of $r$. Denoting with $\dot{M}$ the accretion rate, it follows from the rest mass conservation $\dot{M}=4 \pi r_{S}^{2} c r^{2} \rho y v$, that the gas density scales as $\rho=\rho_{H} r^{-3 / 2}$. Since $y \simeq 1$ in free-fall (hence $v \simeq r^{-1 / 2}$ ), and introducing the accretion rate in units of the Eddington rate $\dot{m}=\dot{M} / \dot{M}_{E}\left(\dot{M}_{E}=L_{E} / c^{2}\right)$, the density at the horizon is related to $\dot{m}$ by $\dot{m}=2 \kappa_{s} \rho_{H} r_{S}$, where $\kappa_{s}$ is the scattering opacity. The expression for the scattering depth in the flow follows immediately and is $\tau_{s}=\int_{r}^{\infty} \kappa_{s} \rho r_{S} d r=2 \kappa_{s} \rho_{H} r_{S} r^{-1 / 2}=\dot{m} r^{-1 / 2}$. The effects of bulk motion Comptonization on the emerging spectrum are governed by the product of the scattering depth times the flow velocity which gives the fractional energy change suffered by a photon undergoing repeated scatterings before escaping (see e.g. Nobili, Turolla \& Zampieri 1993). In the present case $\tau_{s} v=\dot{m} r^{-1}$ and the trapping radius, defined as the locus where $3 \tau_{s} v=1$, is located at $r_{\text {trap }}=3 \dot{m}$

We have computed several sequences of models for $\dot{m}$ in the range $1 \leq \dot{m} \leq 12$. All models include both electron scattering and true emission/absorption. In order to investigate 
the effects of the spatial distribution of the input photons under a minimal set of assumptions, we adopted an artificial opacity coefficient $\kappa_{a, \nu}$ defined as to produce an absorption depth

$$
\tau_{a}= \begin{cases}\tau_{a, H} & r \leq r_{a} \\ \tau_{a, H}\left(\frac{r_{a}}{r}\right)^{n} & r>r_{a}\end{cases}
$$

where $\tau_{a, H}$ is the absorption depth at the horizon and $r_{a}$ and $n$ are adjustable parameters. With the above definition absorption is color-blind, i.e. $\kappa_{a, \nu}=\kappa_{a}$. However, since we retain Kirchhoff's law, the emission coefficient depends on frequency and is given by

$$
j_{\nu}=\tau_{a} r^{-1} B_{\nu}(T)
$$

where $B_{\nu}(T)$ is the Planck function at temperature $T$. We then want to explore the effects of bulk motion Comptonization on the emerging spectrum when $\dot{m}$ is varied, along various sequences of models characterized by different spatial distributions of the emissivity, eq. (3). Particular care must be devoted to the fact that the sequences should be self-similar as far as the role of emission/absorption is concerned. If this is not ensured the spectral behavior may be influenced by the different interplay between scattering and absorption when the accretion rate changes. The self-similarity can be imposed in different ways: here we ask that the scattering and absorption depths become equal at a given radius $r_{c}$ (hereafter the "crossing radius"), which is the same for all models. Using the expression for the absorption depth given by (2) with fixed $n$, the condition $\tau_{s}\left(r_{c}\right)=\tau_{a}\left(r_{c}\right)$ allows to derive $\tau_{a, H}$ for each value of $\dot{m}, \tau_{a, H}=\dot{m}\left(r_{c} / r_{a}\right)^{n-1 / 2} r_{a}^{-1 / 2}$. In our calculations we used $r_{a}=2.5, r_{c}=1.8 r_{a}$ and $n$ in the range $3 \leq n \leq 7$. We note that both $r_{a}$ and $r_{c}$ should be of order unity if the flow has to be scattering dominated up to small radii. Provided this condition is fulfilled, we have checked that varying them does not change the models much.

With this choice of the parameters the absorption depth at $r_{c}$ is always larger than unity for $\dot{m} \geq 1$, so all models have an inner core which is optically thick to true absorption. Beyond $r_{c}$ both the source of seed photons and the true opacity rapidly decay with radius, the distributions becoming sharper with increasing $n$, thus in this region bulk motion Comptonization is the most efficient process. Figure 1 illustrates the dependence of the relevant length-scales on the accretion rate and Figure 2 shows the radial variation of the frequencyintegrated emission coefficient for different values of $n$ and $\dot{m}$. Because of our assumption of self-similarity, upon normalization all curves with the same $\dot{m}$ coincide in Fig. 2. All models have been computed on a radial grid which covers the range $0.2 \leq \log r \leq 6$ and is equally spaced in $\log r$. Although the actual integration of eq. (1) along the rays has been 
carried out on a much finer grid, the radiation intensity was stored for 40 values of the radial coordinate. Since our main goal is to investigate the effects of dynamical Comptonization, we consider here an uniform temperature medium. The exact value of $T$ in unimportant and only fixes the scales of both the photon energy and the intensity (the latter because we have thermal emission). We used 30 energy points, taken to coincide with Gauss-Lobatto quadrature abscissae, in the range $0.3 \leq E / k T \leq 20$, plus additional ten points both below and above the two limits (see Zane, Turolla, Nobili \& Erna 1996 for details). In our scheme the angular resolution is fixed by the number of rays along which the transfer equation is integrated and is not constant along the radial grid. With our present choice of the parameters the minimum number of $\mu$ points (which happens at the photon radius or at the inner boundary if the latter is larger) is 20. Because $\tau_{a}>\tau_{s}>1$ for $r<r_{a}=2.5$ and the absorption opacity in independent on frequency, the spectrum below $r_{a}$ is blackbody (see eq. [3]). For this reason we decided to put the inner boundary just outside the photon radius, at $r_{b} \simeq 1.6$. This speeds up the calculation since the transfer equation needs not to be solved along the trapped photon trajectories. Standard boundary conditions for a non-illuminated

medium have been used: $f\left(r_{b}, \mu>0\right)=B_{\nu}(T) / E^{3}$ and $f\left(r_{\text {out }}, \mu<0\right)=0$, where $r_{\text {out }}$ is the outer boundary of the integration domain.

The emerging spectrum for different $\dot{m}$ is shown in Figure 3 for the three cases $n=$ $3,4,5$. The appearance of a high-energy power-law tail is clearly seen comparing the emergent photon distribution with the blackbody spectrum (the dashed line in Fig. 3). The spectral index depends both on $\dot{m}$ and on the particular sequence of models. The derived values of the photon index $p$ are plotted as a function of $\dot{m}$ for different $n$ in Figure 4 .

\section{Approximated Analytical Solutions}

The numerical results presented in $\S 2$ made evident a dependence of the spectral index on the properties of each particular sequence, at least in the range of $\dot{m}$ that has been spanned. In order to address this point further, we investigate systematically the properties of the analytical solutions presented by TMK97. We then present an application of the method to cases relevant to the models presented in $\S 2$. Although this approach strictly holds only for an isotropic radiation field, we will show that it correctly describes the numerical sequences for $\dot{m}$ not too close to unity and helps understanding the dependence of the numerical models on the various input parameters. 


\subsection{The analytical solution}

By following TMK97, we write the kinetic equation for the angle-averaged photon occupation number $n=n(r, \nu)$ [their eq. (14)] as

$$
\tau \frac{\partial^{2} n}{\partial \tau^{2}}-\left(\tau+\frac{3}{2}\right) \frac{\partial n}{\partial \tau}-\frac{1}{2} x \frac{\partial n}{\partial x}=-\frac{\dot{m}}{2} \frac{j_{\nu}}{\rho \kappa_{s}},
$$

where $\tau=(3 / 2) \dot{m} / r, x=h \nu / k T$. As in TMK97, second order terms in $v$ have been neglected and we restricted to the case in which the source function is the product of a purely spatial part, $S(\tau)$, and a purely energy-dependent part, $g(x)$. Moreover, we did not include thermal Comptonization and treated the scattering as elastic in the electron rest frame.

The eigenfunctions or the space (radial) operator which are well behaved $\left(\sim r^{-2}\right.$ for $r \rightarrow \infty)$ are given by

$$
R_{k}=C \tau^{5 / 2} \Phi\left(-\lambda_{k}^{2}+5 / 2,7 / 2, \tau\right)
$$

where $\Phi(a, b, z)$ is the confluent hypergeometric function (Kummer's function, see Abramowitz $\&$ Stegun 1970), $C$ is a constant and the eigenvalues $\lambda_{k}^{2}$ are the roots of the equation

$$
p \Phi\left(-\lambda_{k}^{2}+\frac{5}{2}, \frac{9}{2}, \tau_{b}\right)+q \Phi\left(-\lambda_{k}^{2}+\frac{3}{2}, \frac{9}{2}, \tau_{b}\right)=0
$$

with

$$
\begin{aligned}
p= & {\left[\frac{5}{2}-\left(2 \lambda_{1}^{2}+\epsilon\right) \frac{\tau_{b}}{3}\right]\left(-\lambda_{k}^{2}+\frac{3}{2}+\tau_{b}\right)+} \\
& \tau_{b}\left(-2 \lambda_{k}^{2}+\frac{1}{2}+\tau_{b}\right) \\
q= & {\left[\frac{5}{2}-\left(2 \lambda_{1}^{2}+\epsilon-3\right) \frac{\tau_{b}}{3}\right]\left(\lambda_{k}^{2}+2\right) ; }
\end{aligned}
$$

here $\epsilon=-3(1-A) \sqrt{r_{b}} /[2(1+A)], \tau_{b}$ and $r_{b}$ are depth and the radius of the inner boundary, respectively, and $A$ is the albedo at $r_{b}$. We note that eq. (6) is just a rearrangement of eq. (A6) of TMK97.

Expanding the spatial part of the source function over the complete set of eigenfunctions $R_{k}$

$$
S(\tau)=\sum_{k=1}^{\infty} c_{k} R_{k}(\tau)
$$


and looking only for separable solutions of the form

$$
n(\tau, x)=R(\tau) N(x)=\sum_{k=1}^{\infty} a_{k} R_{k}(\tau) N_{k}(x),
$$

gives

$$
N_{k}(x)=2 \frac{c_{k}}{a_{k}} x^{-2 \lambda_{k}^{2}} \int_{0}^{x} t^{2 \lambda_{k}^{2}-1} g(t) d t \equiv \frac{c_{k}}{a_{k}} \hat{N}_{k}(x) .
$$

The emergent luminosity is then expressed as

$$
L(\tau=0, x) \propto x^{3} \sum_{k=1}^{\infty} c_{k} \hat{N}_{k}(x)
$$

We note that the coefficients $a_{k}$ do not enter expression (10). The emergent luminosity only depends on the $c_{k}$ 's, i.e. on the spatial distribution of the source function. These coefficients may be readily evaluated as integrals of $S(\tau)$ times a weighting function (see TMK97, Appendix B). The source function corresponding to the numerical models presented in $\S 2$ is $S(\tau) \propto \tau^{n-1 / 2}$ for $r \geq r_{a}$ where scattering is dominant.

In order to investigate the behavior of the spectral index, we evaluated $L(\tau=0, x)$ in the range $0.1 \leq x \leq 500$, for different values of $\tau_{b}$ and $n=3,4,5$, assuming that $g(x) \propto[\exp (x)-1]^{-1}$ (i.e. the primary photon spectrum is blackbody). We use here the same notation as in TMK97, where the eigenvalues were labeled according to their asymptotic value at $\dot{m} \gg 1$. In particular, the first eigenvalue $\lambda_{1}^{2}$ is the smallest root of eq. (6). Since the different roots never cross each other when $\dot{m}$ decreases, the hierarchy of the eigenvalues can be derived from their asymptotic values at large $\tau_{b}$. As shown by TMK97, in this limit the roots are

$$
\begin{aligned}
& s_{1} \sim \frac{3}{2}+\frac{3}{4}\left(\frac{1-A}{1+A}\right) \sqrt{r_{b}}, \\
& s_{k} \sim \frac{2 k+1}{2}, \quad k \geq 2
\end{aligned}
$$

out of which only the smallest one is meaningful and should be then inserted back into eq. (6) to compute the $\lambda_{k}^{2}$ 's with $k \geq 2$. As the previous expressions shows, it is $\lambda_{1}^{2}=s_{1}$ if $3 / 2+(3 / 4)(1-A)(1+A)^{-1} \sqrt{r_{b}}<5 / 2$ while $\lambda_{1}^{2}=s_{2}$ otherwise. Once eq. (6) is solved with 
the appropriate value for $\lambda_{1}^{2}$, the two sequences of the eigenvalues at large $\tau_{b}$ turn out to be

$$
\begin{aligned}
& \lambda_{1}^{2} \sim \frac{3}{2}+\frac{3}{4}\left(\frac{1-A}{1+A}\right) \sqrt{r_{b}}, \\
& \lambda_{k}^{2} \sim \frac{2 k+1}{2}, \quad k \geq 2
\end{aligned}
$$

for $3 / 2+3 / 4(1-A) /(1+A) \sqrt{r_{b}}<5 / 2$ and

$$
\lambda_{k}^{2} \sim \frac{2 k+3}{2} \quad k \geq 1
$$

for $3 / 2+(3 / 4)(1-A)(1+A)^{-1} \sqrt{r_{b}}>5 / 2$. Since $\hat{N}_{k}(x) \sim x^{-2 \lambda_{k}^{2}}$ for $x \gg 1$, the flatter spectral mode corresponds to the smaller $\lambda_{k}^{2}$.

It is natural to expect that terms of higher order do not contribute significantly to the series (10) at large frequencies. Naively, one might be tempted to conclude that the value of the spectral index in the power law tail is only dictated by the smallest eigenvalue (the same conclusion is in fact at the basis of most previous investigation, see e.g. TMK97). However, while terms with $k>2$ never significantly contribute to the high-energy tail, the dominant mode can be either the first or the second, depending on the parameters of the model. For $\lambda_{1}^{2} \rightarrow 5 / 2$ the first mode is always the dominant one, but when $\lambda_{1}^{2} \rightarrow$ $3 / 2+(3 / 4)(1-A)(1+A)^{-1} \sqrt{r_{b}}<5 / 2$, it dominates only in a limited range of relatively small $\dot{m}$. This is illustrated in Figure $5 \mathrm{a}$, b, which shows the first nine terms of the series (10) for $A=0, r_{b}=1, n=5$ and two different values of $\dot{m}=2 \tau_{b} r_{b} / 3$. At relatively low $\dot{m}$ (Fig. 5a) the mode $k=1$ is indeed the dominant one, but when $\dot{m}$ increases (Fig. 5b) it is the $k=2$ term which gives the larger contribution over many decades in frequency. The main reason is that the expansion coefficient $c_{1}$ goes to zero exponentially fast when $\tau_{b}$ increases. This has been already noted by TMK97 (see also Mastichiadis \& Kylafis 1992) in discussing the equivalence of their solution to that of Payne \& Blandford (1981) for $\tau_{b} \rightarrow \infty$. However they did not point out that, as a consequence, the eigenvalue $\lambda_{1}^{2}$ does not represent the spectral index at large $\dot{m}$. The main consequence is the appearance of a range in $\dot{m}$ over which the spectral index makes a "transition" from the first to the second mode. This is illustrated in Figure. 6 which makes evident that the spectral index in the transition region do depend on the spatial distribution of the source. We stress that all these considerations do not reflect the behavior of the power-law tail in the limit $x \rightarrow \infty$. At really large energies, the spectral index is always fixed by $\lambda_{1}^{2}$, as it can be guessed from Figure 5b. However, the observationally accessible part of the high-energy tail is indeed dictated by $\lambda_{2}^{2}$.

Figure 7a illustrates the dependence of the spectral index on $r_{b}$. As it can be seen, the variation with $r_{b}$ is not monotonic. At low $\dot{m}$ spectra tend to be harder as the inner boundary moves closer to the horizon, while at larger $\dot{m}$ the behavior of the spectral index is 
more complicated. Models with different $r_{b}$ approach $2 \lambda_{2}^{2}-2$ in correspondence of different values of $\dot{m}$, and beyond $\dot{m} \approx 10$ spectra might be softer at smaller $r_{b}$. It is also worth to stress that, since $\dot{m}=2 \tau_{b} r_{b} / 3$ and diffusion approximation holds for $\tau_{b}>1$, the larger is $r_{b}$ the larger is the value of $\dot{m}$ at which the analytical solution is not trustworthy any more. The dependence on the albedo $A$ is shown in Figure 7b. Again, all spectral indices tend toward a common asymptotic value which is constant and is given by the second mode. However, before this limit is reached, the dependence on $A$ is monotonic. The larger is $A$ at the inner boundary, the harder is the emergent spectrum, in agreement with previous findings (see Mastichiadis \& Kylafis 1992 and TMK97).

\subsection{Comparison with numerical models}

We are now in the position to reconsider the behavior of the spectral index in the numerical sequences in the light of the results discussed in the previous section. The overall situation described by the numerical models is, of course, different from that assumed in the analytical solution, in particular because in the former a) the flow has an inner core, optically thick to true emission/absorption, b) true absorption is consistently accounted for through Kirchhoff's law, and c) relativistic effects are correctly included.

The first consequence is that the numerical sequences we have computed are far from being evaluated at a given, fixed value of both $r_{b}$ and $A$. However, a comparison between numerical and analytical models can be attempted, assuming that $r_{b}$ coincides with the boundary of the region in which scattering is the main source of opacity, $r_{a b s}$, where the absorption depth equals unity [see $\S 2$ and eq. (2)]. In the region between $r_{a}$ and $r_{a b s}$ the role of true absorption is certainly non-negligible and affects the emerging spectrum together with Comptonization. We stress that this is an oversimplification, since it is not possible to define an inner boundary in our numerical models in the same way as it was done in $§ 3.1$. This implies also that the value of the albedo at the inner boundary is ill-defined. On the other hand, in all our models the region $r \lesssim r_{a b s}$ is optically thick to absorption and is at constant temperature. Under these conditions, we expect the ingoing flux at the boundary to be substantially larger than the outgoing one. This amounts to say the inner part of the flow is illuminated from above and the value of the albedo should consequently be small, $A \lesssim 0.1$

In Figure 8 we show the comparison between numerical and analytical sequences, the latter computed taking $r_{b}=r_{a b s}(\dot{m})$, while $A$ is constant along each sequence. For $\dot{m} \gtrsim 4$ the results derived in diffusion approximation are in very good agreement with those computed with the full angle-dependent code for reasonable values of the albedo. As expected, the 
agreement becomes worse at low $\dot{m}$ where the diffusion approximation starts to break down.

\section{Discussion and Conclusions}

Previous investigations on bulk motion Comptonization studied the properties of the emerging spectrum, in particular as far as the formation of a hard, power-law tail is concerned. In this respect, two issues are of the highest relevance in connection with observations of Galactic X-ray binaries (XRBs) in the high/soft state. The first is whether the appearance of a high energy power-law tail is a ubiquitous feature of converging accretion flows, the second is to which extent the spectral index is independent on the details of model, mainly on the spatial and energy distribution of the seed photons. TMK97, Titarchuk \& Zannias (1998) and Laurent \& Titarchuk (1999) have shown that, for the cases they have examined, a power-law tail is always present and the spectral index is almost insensitive to source distribution. Our present results strengthen these conclusions. At the same time, we further clarify the properties of the spectral index and its dependence on the detail of the source.

The main conclusion we derived in $§ 3.1$ (see in particular Fig. 6) is that the photon index $\Gamma$ switches from $\Gamma=2 \lambda_{1}^{2}-2$ to $\Gamma=2 \lambda_{2}^{2}-2$ as the accretion rate increases. The location and width of the transition region depends in general on the boundary conditions, i.e. on $r_{b}$ and $A$ (see e.g. Fig. 7b). For example, for the case $r_{b}=1, A=0$, the transition occurs for $5 \lesssim \dot{m} \lesssim 15$ while in solutions with larger $r_{b}$ the transition region is wider and shifted toward larger $\dot{m}$. The properties of the spectral index in the different ranges of $\dot{m}$ (below, within and above the transition region) are dramatically different, as it is shown by both numerical and analytical results.

We now discuss this point in more detail by taking as representative the case $r_{b}=1$, $A=0$, illustrated in Figure 6 . As we can see, for low accretion rates, $\dot{m} \lesssim 5$, the photon index $\Gamma$ is indeed independent on the source spatial distribution and is related only to the first eigenvalue, $\Gamma=2 \lambda_{1}^{2}-2$. Although $\lambda_{1}^{2}$ does depend on both the albedo and the location of the inner boundary, its value is always the same for a flow which is absorption thin all the way down to the horizon. This is likely to reproduce the situation in a black hole XRB, in which most of the material is carried inwards by a standard disk and only a small fraction accretes roughly spherically. The different behavior of our numerical models is not in contradiction with this statement. In fact, an important point to realize is that, owing to the presence of an absorption thick core, the range of $\dot{m}$ explored numerically falls below (or at most at the beginning of) the transition region. The value of the photon index (which, in this range, is dominated by $\lambda_{1}^{2}$ ) changes because of a change in both the location of the inner boundary 
$\left(r_{a b s}\right.$ is a function of $\left.\dot{m}\right)$ and the albedo. Therefore, the variation of $\Gamma$ with $n$ (i.e. with the photon source) seen in our numerical sequences is not directly connected with the change of the source itself, but is largely due to the change in the boundary conditions.

The situation is different in the transition region, i.e. for intermediate values of the accretion rate $\left(5 \lesssim \dot{m} \lesssim 15\right.$ in the case $r_{b}=1$ and $\left.A=0\right)$. Now the spectral index genuinely depends on the spatial distribution of primary photons. This is true irrespective of the assumed boundary conditions, i.e. all models are expected to exhibit a range in $\dot{m}$ (which can vary depending on $r_{b}$ and $A$ ) over which the shape of $S(\tau)$ is important in determining $\Gamma$ (see Fig. 7b). It should be noted, nevertheless, that $\Gamma$ depends rather weakly on the spatial distribution, as it can be seen from Fig. 6. As a consequence, spatially concentrated photon sources (like the ones we used here) or more diffused ones (as that employed by TMK97) give raise to spectral indices which are not sensibly different. This implies that $\Gamma$ is about the same for primary photons produced in the disk (diffused source) or in the inner, denser region of an $\mathrm{ADAF}$ (concentrated source).

For large accretion rates (i.e. $\dot{m} \gtrsim 15$ for $r_{b}=1$ and $A=0$ ), the spectral index quickly approaches $\Gamma=2 \lambda_{2}^{2}-2$ and becomes again independent on the source distribution. Since the value of $\dot{m}$ at which the transition from $2 \lambda_{1}^{2}-2$ to $2 \lambda_{2}^{2}-2$ is completed is larger than the value at which $\lambda_{2}^{2}$ attains its asymptotic value $(\dot{m} \sim 10)$, the index is also insensitive to both $r_{b}$ and $A$, and it is $\Gamma \sim 3$. The same considerations apply to solutions with larger $r_{b}$ (see Fig. 7b).

We turn now to the comparison of present results with those derived in previous investigations for the spectral index and its dependence of the accretion rate. Here we are concerned only with the case of $A=0$ and $r_{b}=1$, that is to say we assume that the accreting object is a black hole and true emission/absorption can be neglected. The results are summarized in Fig. 9, where the spectral index is shown as a function of the accretion rate. As it can be seen, the major differences are at low values of $\dot{m}$ where the various approaches give extremely different results. We note first that methods based on the solution of the full (angle-dependent) transfer problem provide quite similar (albeit not exactly equal) results with $\Gamma$ a monotonically decreasing function of $\dot{m}$. Relativistic diffusion approximation predicts much softer spectra and $\Gamma$ is now monotonically increasing with the accretion rate. Non-relativistic diffusion produces somehow an intermediate result in which the spectral index exhibits a minimum.

An obvious point to recall is that diffusion-limit solutions are valid only for $\tau \gg 1$, so they are bounded to fail when $\dot{m} \sim \tau_{b} \geq \tau$ is a few or less. Moreover, the relativistic analysis by Turolla, Zane, Zampieri \& Nobili (1996) strictly speaking applies only below the trapping radius. Clearly, for small accretion rate the right answer is that provided by the 
full transfer calculation. However, the comparison of the various curves in Fig. 9 shows that the different behavior of the index at small $\dot{m}$ is not a direct effect of general relativity and hence of the presence of the horizon. Would this be true one should expect the relativistic diffusion approximation to describe much better the solution. Also, in free-fall gravity and dynamics cancel each other at a given radius, so no relativistic effects are expected in the flow local rest frame. This argument is not against the claim that the detection of a powerlaw tail with index $\sim 2.5-3$ is indicative of a black hole rather than a neutron star. The reason for this, nevertheless, are not general relativistic effects but the fact that only a black hole provides the conditions under which a converging flow may reach $\tau_{b} \gtrsim 1$. This is not possible for a neutron star, because of the much higher accretion efficiency. The presence of a solid crust at $\sim 3 r_{g}$ implies that all the kinetic energy of the inflowing material is released upon impact with an efficiency $\approx 1 / 6$. This means that $\dot{m} \sim 1$ is enough to produce a near-Eddington luminosity which slows down the flow until a settling solution is established (see e.g. Miller 1990; Zampieri, Turolla \& Treves 1993). As a consequence, $\tau_{b}^{N S} \ll \tau_{b}^{B H}$ at the same $\dot{m}$ because the velocity is much smaller. Increasing $\dot{m}$ is of no avail since, as Zampieri, Turolla \& Treves (1993) have shown, $\tau_{b}$ reaches $\sim 1$ for $\dot{m} \sim 3.5$ then starts to decrease. Consequently, for a spherically accreting neutron star a power-law tail may indeed form but it is always steeper (softer spectrum) than in black holes. It should be realized also that the inner part of the accreting flow, close enough to the star crust, is bound to be effectively thick, so assuming that the surface acts as a reflecting boundary does not appear entirely realistic.

Finally, a comment of the asymptotic value of the index for $\tau_{b} \rightarrow \infty$ is in order. Both relativistic and non-relativistic diffusion calculations give an asymptotic index $\Gamma=3$. The fact that the relativistic curve of Turolla, Zane, Zampieri \& Nobili (1996) approaches 3 at much larger values of $\dot{m}$ is related to the particular source function they used. In fact, monochromatic photons were assumed to be injected at a single radius very close to the horizon. The behavior of the index, as discussed previously, depends on the spatial distribution of the source and the transition region is wider and shifted at larger $\dot{m}$ as the source is more and more concentrated. This shows once more that there are no substantial differences between relativistic and non-relativistic diffusion, once proper allowance for the spatial distribution of seed photons is made.

In concluding, we would like to stress that present results can not be directly compared with observations. As several investigations have shown (e.g. Shrader \& Titarchuk 1998; Shrader \& Titarchuk 1999; Borozdin et al. 1999), modeling the X-ray spectrum of black hole binaries requires the inclusion of both thermal and dynamical comptonization. Moreover, we neglected electron recoil (e.g. we assumed elastic scattering in the electron rest frame), so no high-energy cut-off is present in our models. If, as it seems reasonable, the estimates 
derived by TMK97 and Laurent \& Titarchuk (1999) apply also in the present case, a cut-off at energies $\approx 300-400 \mathrm{keV}$ is expected. The lack of a break in high-energy spectra of black hole sources may be suggestive of an alternative origin of the power-law tails, e.g. comptonization by thermal/non-thermal electrons (e.g. Gierliński et al. 1999). Up to now, however, observations are not compelling in this respect (e.g. Zdziarski et al. 2001 and Titarchuk \& Shrader 2002 for OSSE observations of GRS 1915+105). Also, Laurent \& Titarchuk (2001) found a high photon compactness near the horizon due to relativistic ray bending. This should lead to pair production and ultimately to an extension of the powerlaw tail to energies $\approx 1 \mathrm{MeV}$. They have also shown that the power-law part of the spectrum at energies less than $\approx 100-200 \mathrm{keV}$ is not affected by nonlinear photon-electron interactions.

\section{Acknowledgments}

Work partially supported by the Italian Ministry for Education, University, and Re-

search (MIUR) through grant COFIN-2000-MM02C71842. LT is grateful to Kinwah Wu for fruitful discussions during his visit to MSSL.

\section{REFERENCES}

Abramowitz M., \& Stegun I.A. 1970, Handbook of Mathematical Functions (Dover: New York)

Borozdin K., et al. 1999, ApJ, 517, 367

Chakrabarti S.K., \& Titarchuk L. 1995, ApJ, 455, 623

Colpi M. 1988, ApJ, 326, 233

Cowsik R., \& Lee M.A. 1982, Proc. R. Soc. London, Ser. A, 383, 409

Ebisawa K., Titarchuk L., \& Chakrabarti S.K. 1996, PASJ, 48, 59

Gierliński M. et al. 1999, MNRAS, 309, 496

Laurent P., \& Titarchuk L. 1999, ApJ, 511, 289

Laurent P., \& Titarchuk L. 2001, ApJ, 562, L67

Mastichiadis A. \& Kylafis N.D., 1992, ApJ, 384, 136 
Miller G.S. 1990, ApJ, 356, 572

Narayan R., Mahadevan R., \& Quataert E. 1999, in The Theory of Black Hole Accretion Disks, Abramowicz M.A., Bjornsson G. and Pringle J.E. eds. (Cambridge University Press: Cambridge)

Nobili L., Turolla R., \& Zampieri L. 1993, ApJ, 404, 686

Papathanassiou H., \& Psaltis D. 2001, preprint (astro-ph/0011447)

Payne D.G., \& Blandford R.D. 1981, MNRAS, 196, 781

Schneider P., \& Bogdan T.J. 1989, ApJ, 347, 496

Shakura N.I., \& Sunyaev R. 1973, A\&A, 24, 337

Shrader C., \& Titarchuk, L. 1998, ApJ, 499, L31

Shrader C., \& Titarchuk, L. 1999, ApJ, 521, L121

Sunyaev R., \& Titarchuk, L. 1985, A\&A, 143, 374

Sunyaev R., \& Titarchuk, L. 1980, A\&A, 86, 121

Titarchuk L., Mastichiadis A., \& Kylafis N.D. 1996, A\&AS, 120, 171

Titarchuk L., Mastichiadis A., \& Kylafis N.D. 1997, ApJ, 493, 863 (TMK97)

Titarchuk L., \& Shrader C. 2002, ApJ, 567, 1057

Titarchuk L., \& Zannias, T. 1998, ApJ, 493, 863

Turolla R., Zane S., Zampieri L., \& Nobili L. 1996, MNRAS, 283, 881

Zampieri L., Turolla R., \& Treves A. 1993, ApJ, 419, 311

Zane S., Turolla, R., Nobili L., \& Erna M. 1996, ApJ, 466, 871

Zdziarski A., et al. 2001, ApJ, 554, L45 


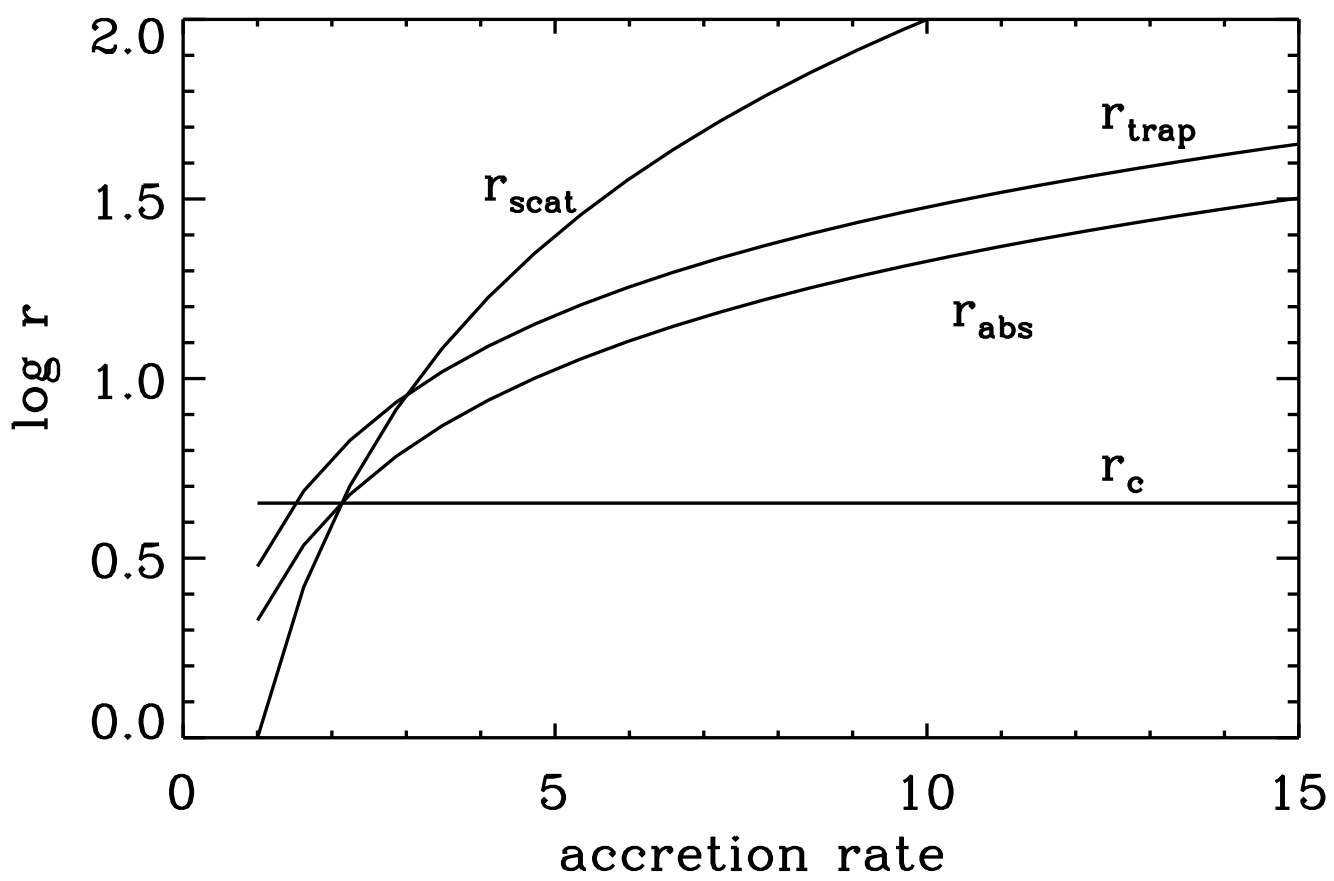

Fig. 1. - The trapping radius $r_{\text {trap }}$, the crossing radius $r_{c}$ and the scattering/absorption photospheric radii, $\tau_{s}\left(r_{\text {scat }}\right)=\tau_{a}\left(r_{a b s}\right)=1$, for different $\dot{m}$ and $n=4$. 


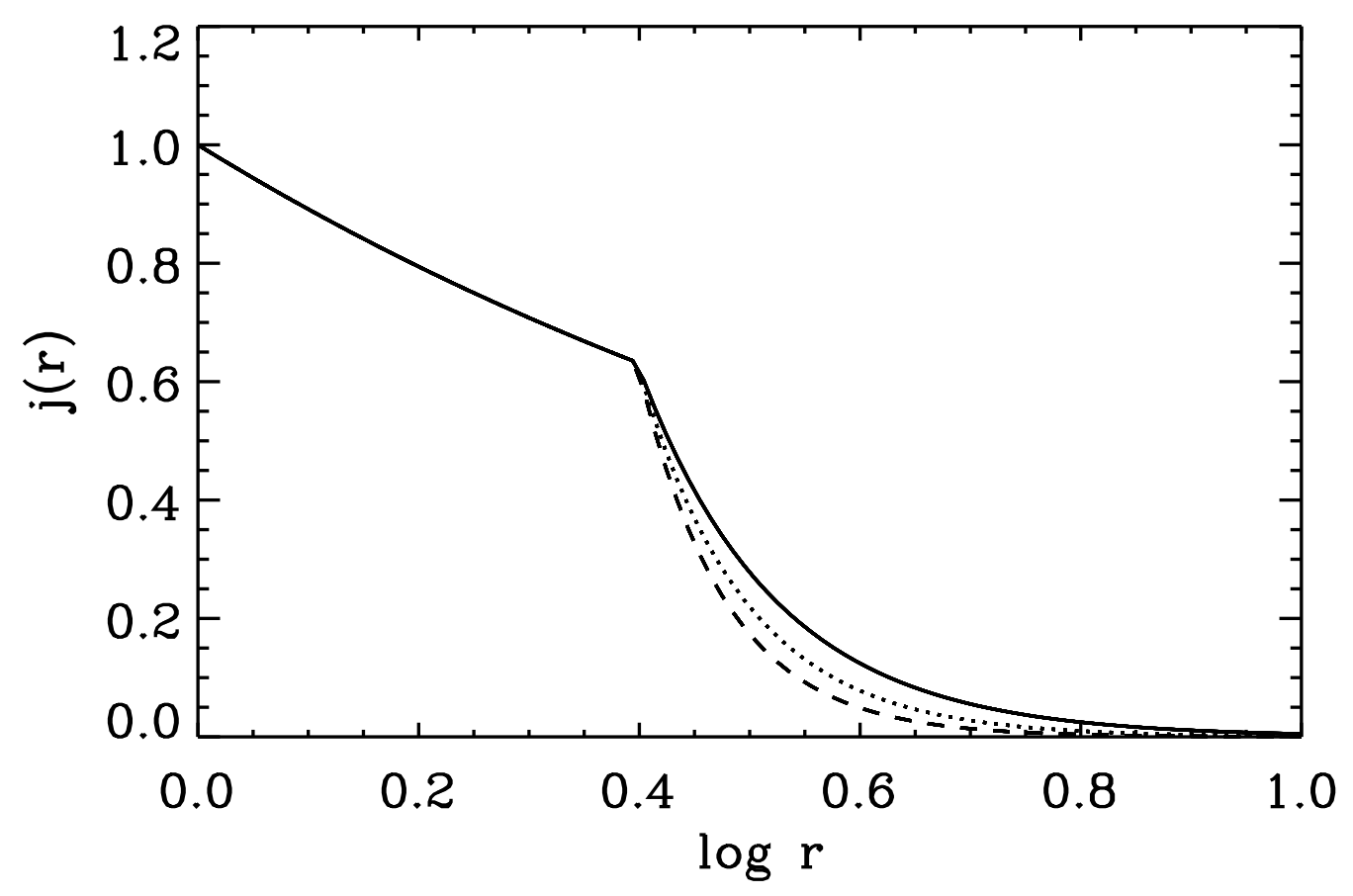

Fig. 2.- The radial dependence of the frequency-integrated emissivity for $n=3$ (full line), $n=4$ (dotted line) and $n=5$ (dashed line); $j(r)$ has been normalized to its value at the inner radial point. 

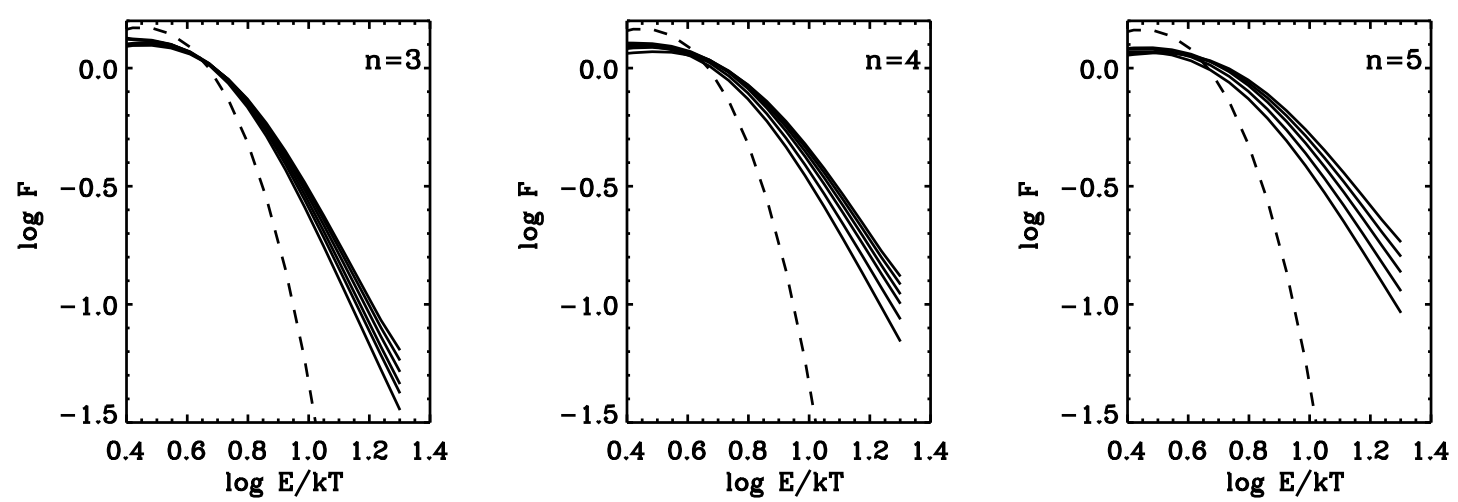

Fig. 3.- The emerging spectrum for $n=3,4,5$ and different values of $\dot{m}$. In each panel $\dot{m}$ increases step 1 from bottom to top curve. Only the high-energy part of the spectra is shown to visualize better the power-law tail. The dashed line is the blackbody spectrum. Flux is in arbitrary units and spectra have been normalized so that they coincide at the lowest energy point (not shown).

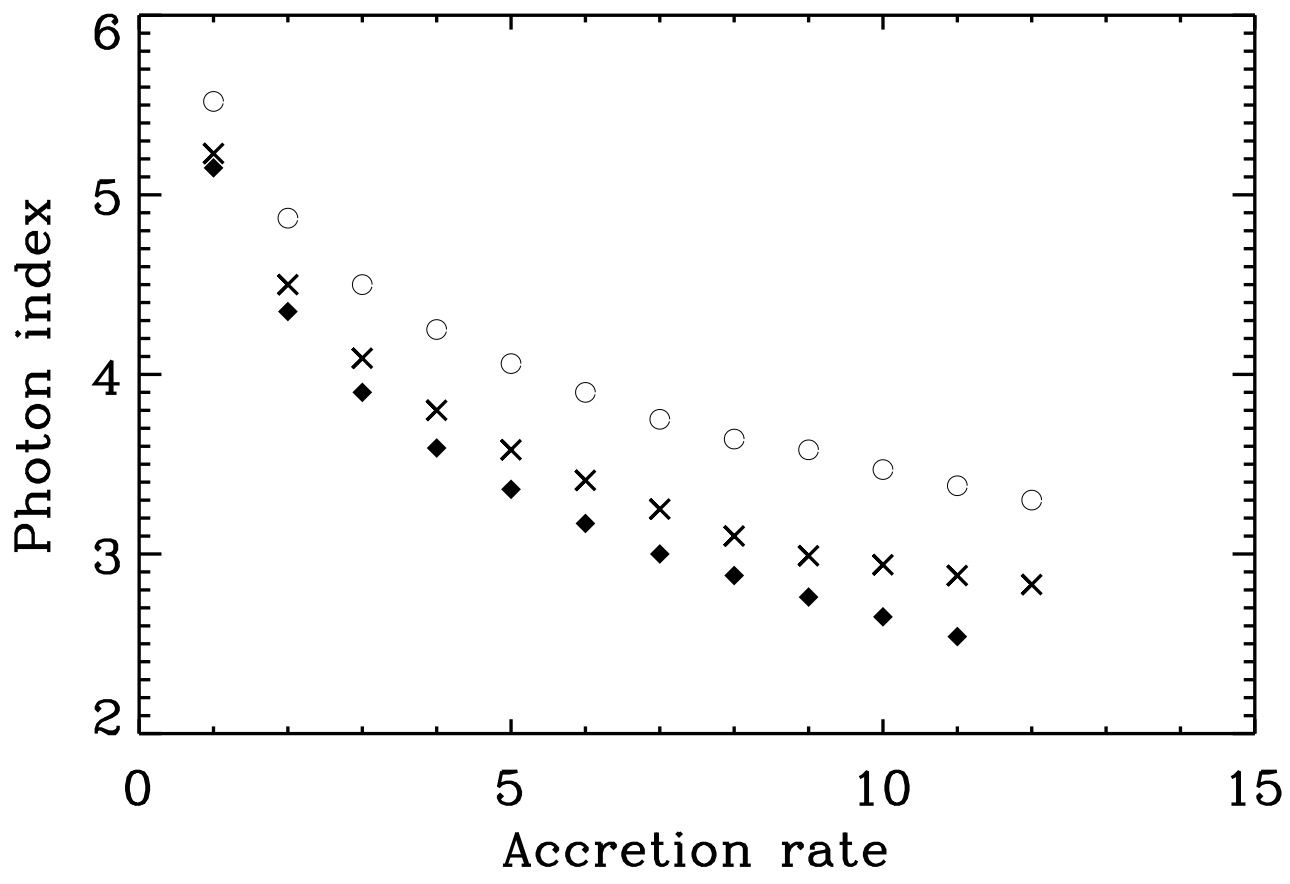

Fig. 4. - The photon index as a function of the accretion rate for the different models discussed in the text: $n=3$ (circles), $n=4$ (crosses) and $n=5$ (diamonds). 

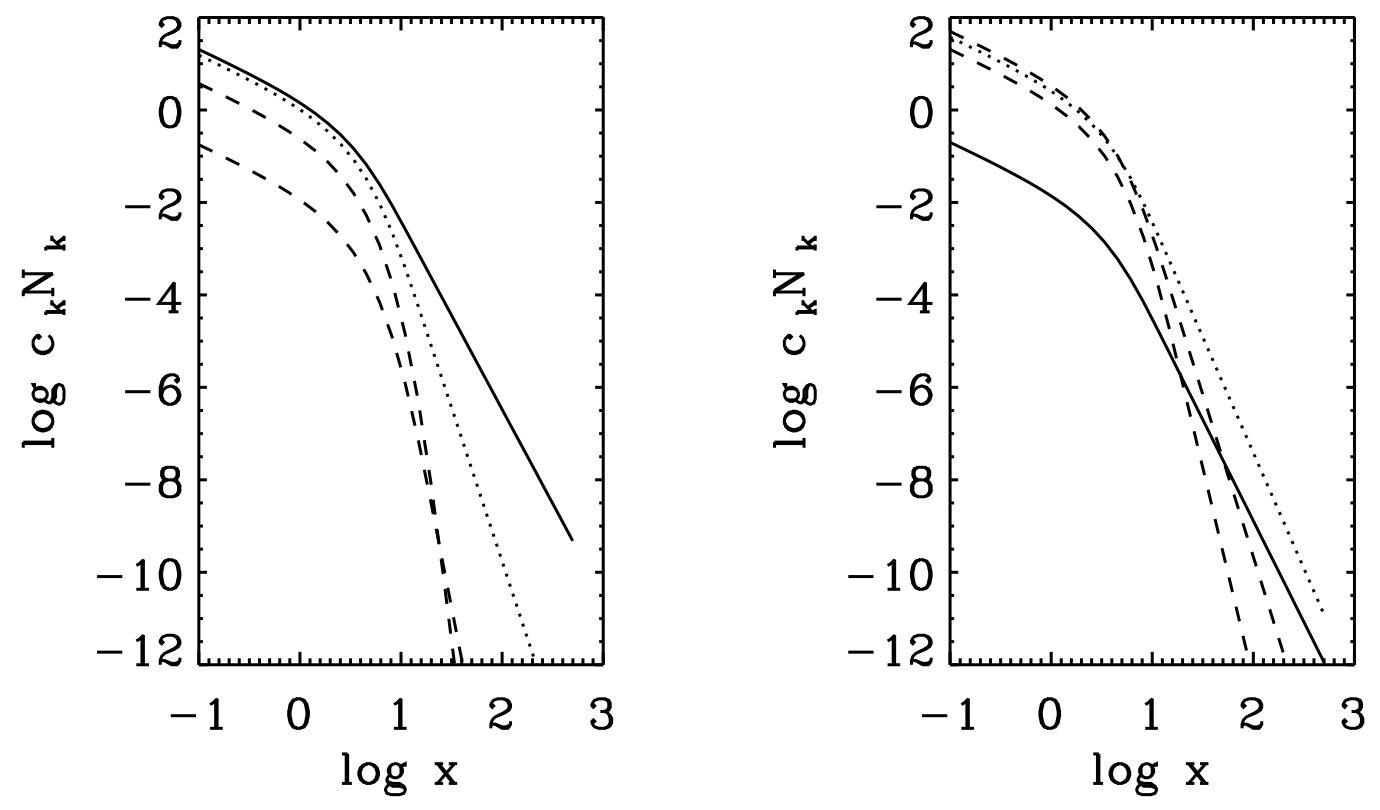

Fig. 5. - The first nine terms of the series for the emergent flux vs. energy for $A=0, r_{b}=1$, $n=5$ and: a) $\tau_{b}=6(\dot{m}=4$, left panel $) ;$ b $) \tau_{b}=20(\dot{m}=13$, right panel $)$. The full line corresponds to $k=1$, the dotted line to $k=2$ and the dashed lines to $k=3, \ldots, 9$, (from top to bottom). 


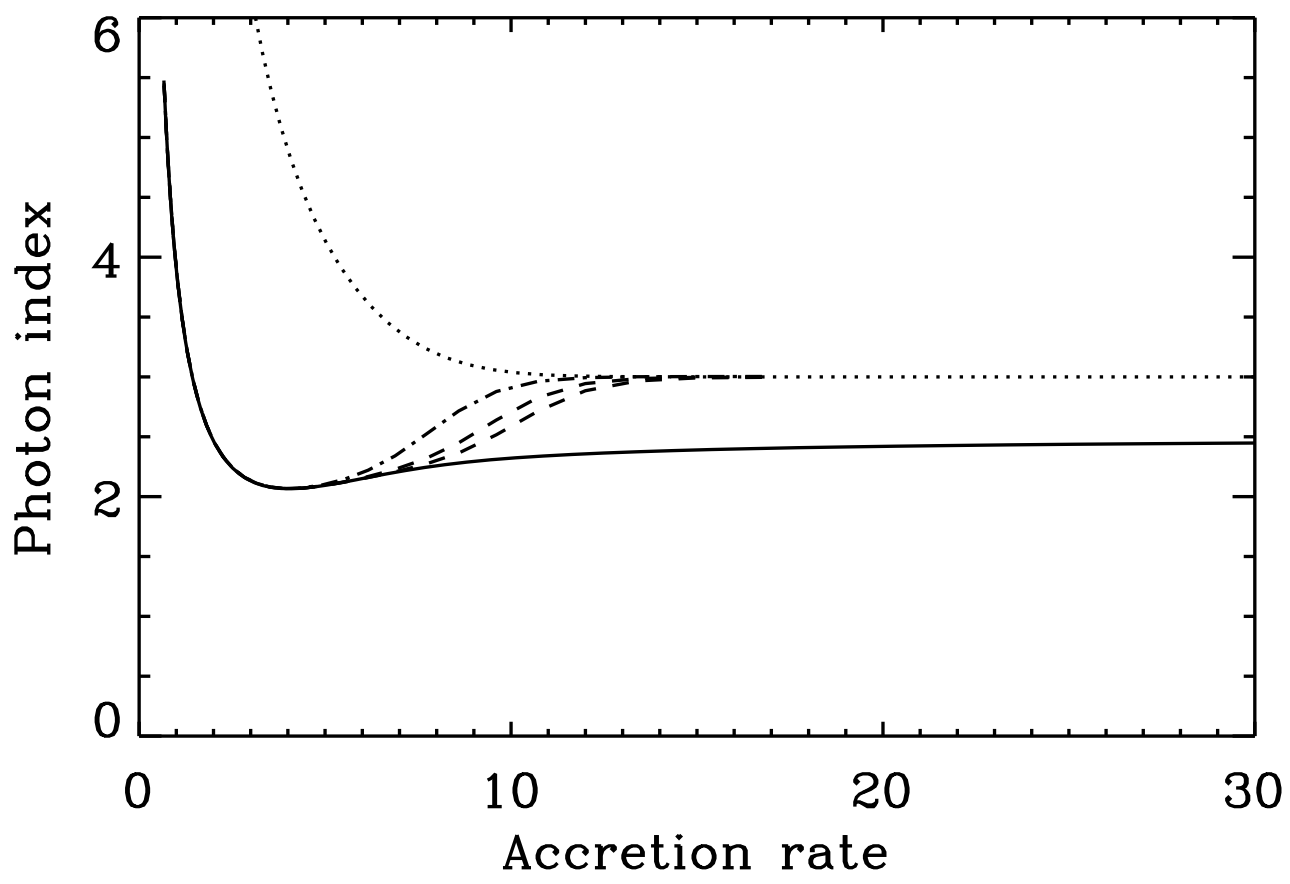

Fig. 6. - The spectral index, derived from eq. (10), vs. $\dot{m}$ : dashed lines are for $n=4$ (upper curve) and $n=5$ (lower curve), the dash-dotted line corresponds to TMK97 source distribution [their eq. (21)]. The full and dotted lines represent $2 \lambda_{k}^{2}-2$ for $k=1$ and $k=2$, respectively. The presence of a transition region where the spectral index switches from the first to the second eigenvalue is evident; here $A=0$ and $r_{b}=1$. 

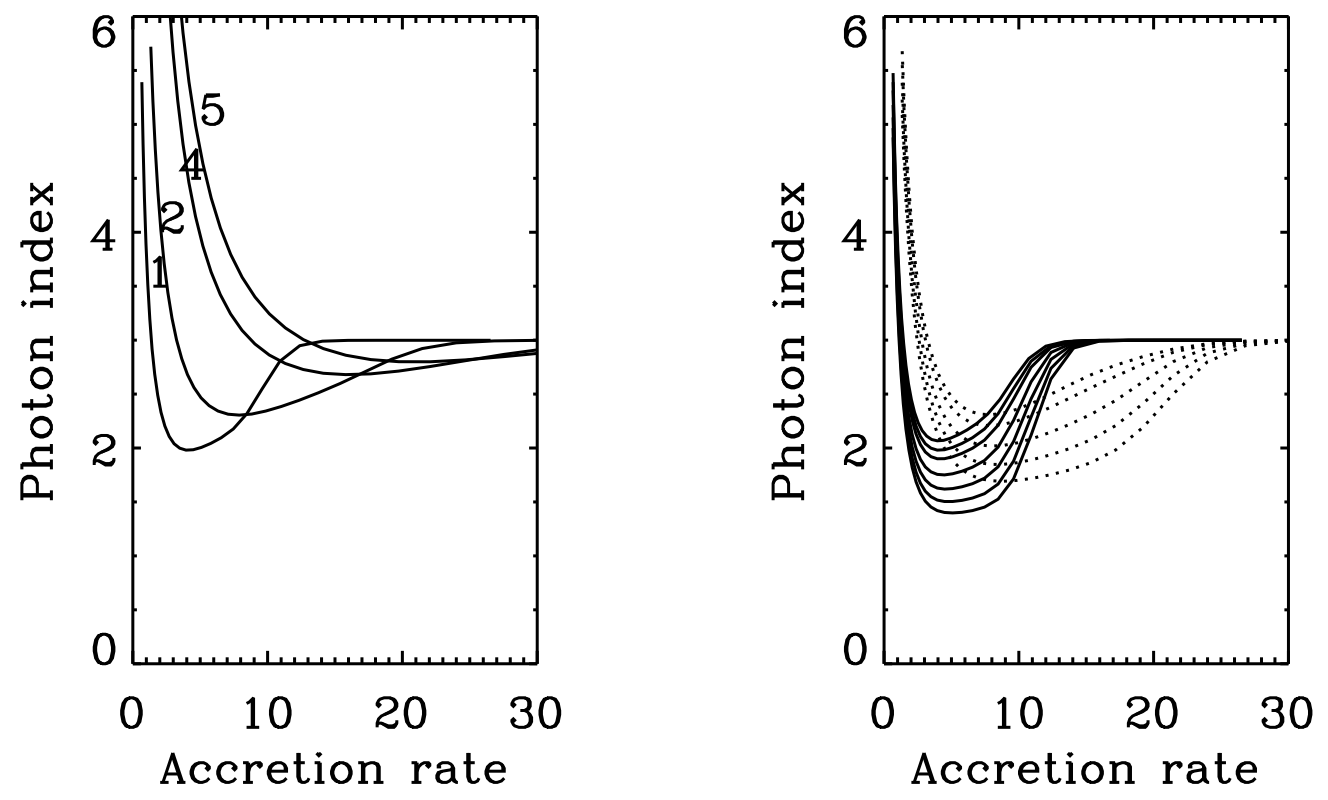

Fig. 7. - The spectral index vs. $\dot{m}$ for $n=4$ : a) different values of $r_{b}$ (the labels on the curves) and $A=0.05$, (right panel); b) different values of $A$ and two values of $r_{b}$ (right panel); here $A=0,0.05,0.1,0.2,0.3,0.4,0.5\left(r_{b}=1\right.$, full lines, from top to bottom) and $A=0.05,0.1,0.2,0.3,0.4$ ( $r_{b}=2$, dotted lines, from top to bottom). 


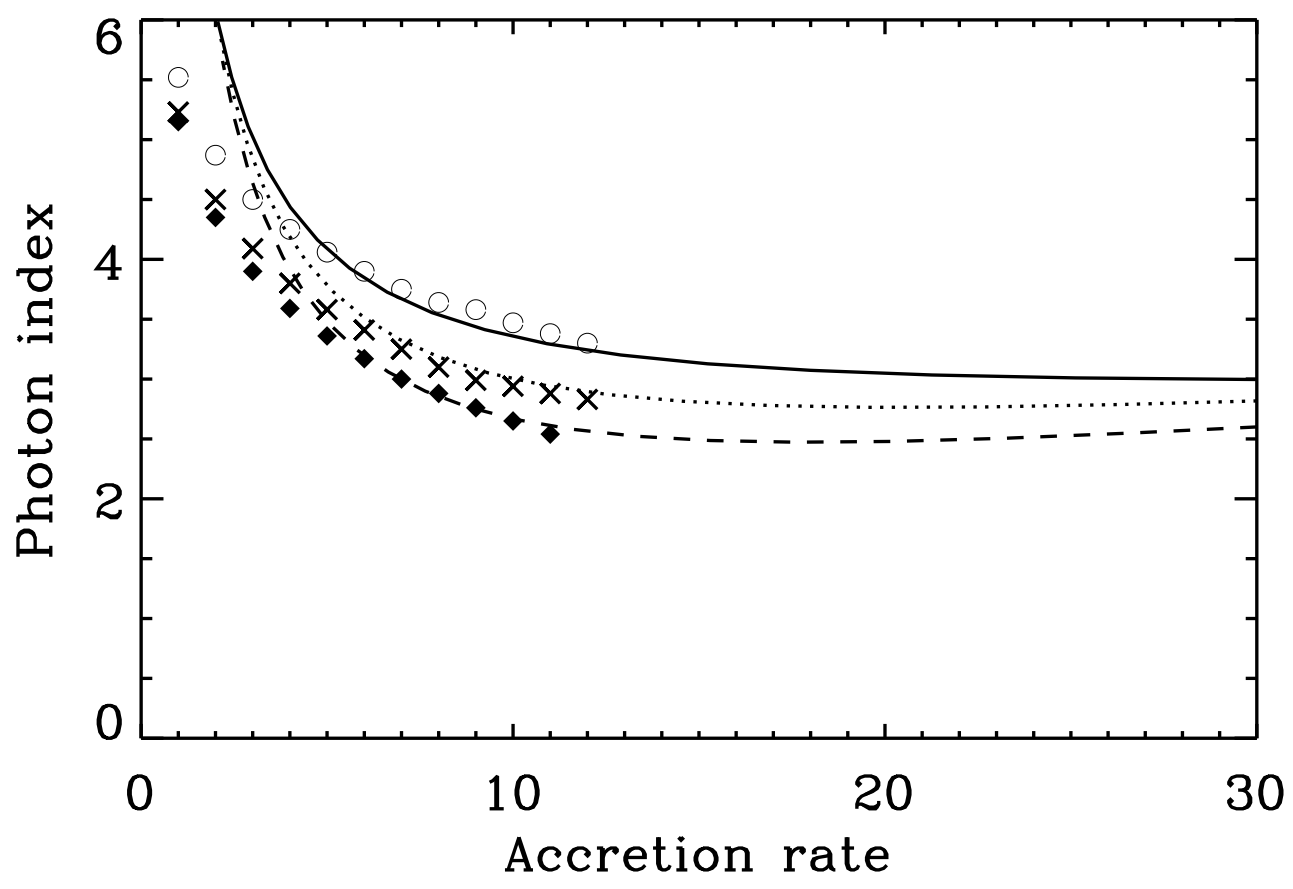

Fig. 8. - The photon index for the numerical sequences discussed in $\S 2$ compared with the analytical estimate from eq. (10) with $r_{b}=r_{a b s}$ for: $n=3, A=0.05$ (full line), $n=4$, $A=0.1$ (dotted line), $n=5, A=0.2$ (dashed line). 


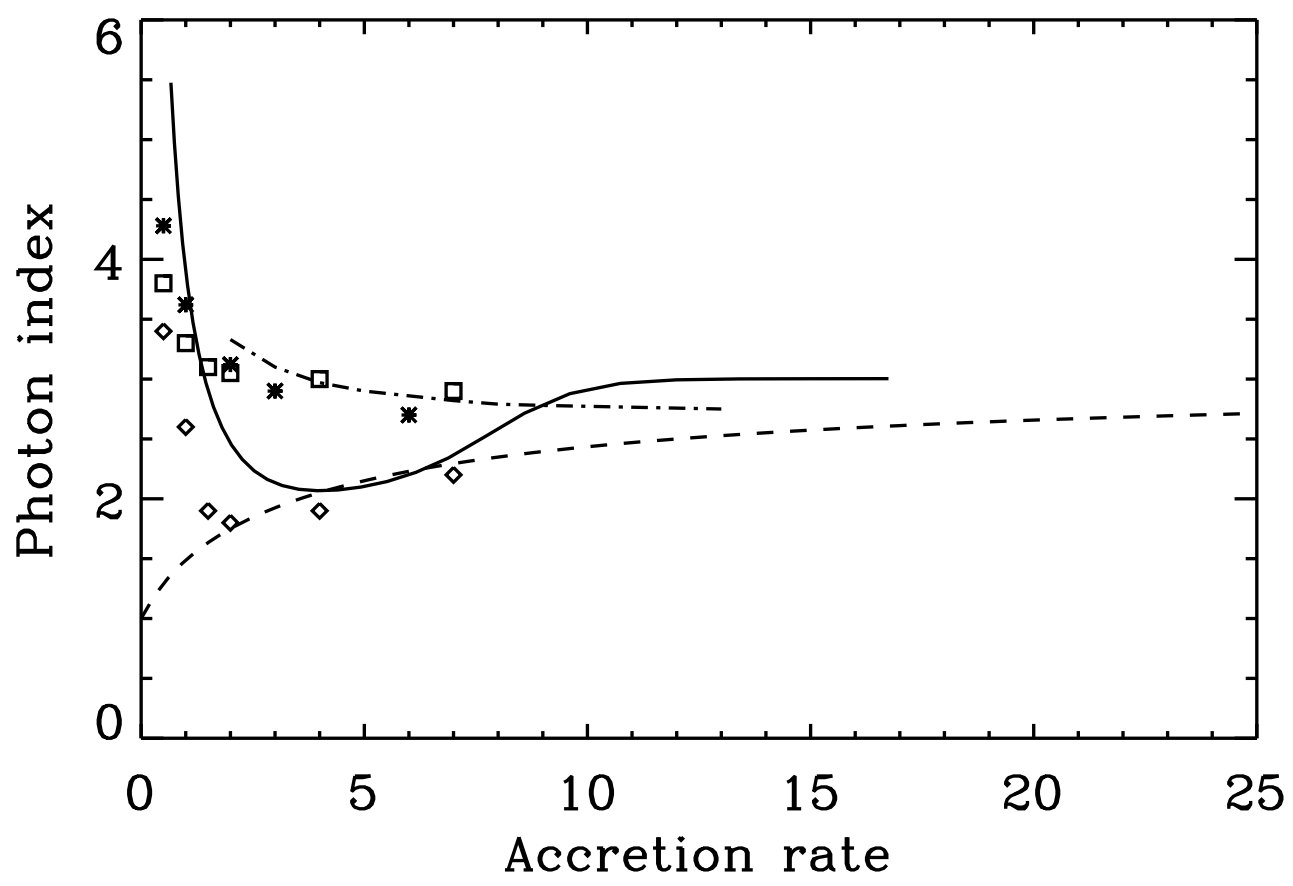

Fig. 9.- The photon index vs. $\dot{m}$ as derived from different studies: non-relativistic diffusion approximation (present investigation, full line), Titarchuk \& Zannias (dash-dotted line), relativistic diffusion approximation (Turolla et al., dashed line), Papathanassiou \& Psaltis (crosses), Laurent \& Titarchuk (Schwarzschild geometry, open squares; flat geometry, diamonds 